Pacific

Journal of

Mathematics

\title{
STABLE LAWS ARISING FROM HITTING DISTRIBUTIONS OF PROCESSES \\ ON HOMOGENEOUS TREES \\ AND THE HYPERBOLIC HALF-PLANE
}

Paolo Baldi, Enrico Casadio Tarabusi, and Alessandro

Figà-TaLamanca 


\title{
STABLE LAWS ARISING FROM HITTING DISTRIBUTIONS OF PROCESSES ON HOMOGENEOUS TREES AND THE HYPERBOLIC HALF-PLANE
}

\author{
Paolo Baldi, Enrico Casadio Tarabusi, and Alessandro \\ FigÀ-TALAMANCA
}

The projective line with respect to a local field is the boundary of the Bruhat-Tits tree associated to the field, much in the same way as the real projective line is the boundary of the upper half-plane. In both cases we may consider the horocycles with respect to the point at infinity. These horocycles are exactly the horizontal lines $\{y=a\}$ with $a>0$ in the real case, while in the case of a local field the horocycles may be thought of as discretizations of the field obtained by collapsing to a point each ball of a given radius.

In this paper we exploit this geometric parallelism to construct symmetric $\alpha$-stable random variables on the real line and on a local field by completely analogous procedures. In the case of a local field the main ingredient is a drifted random walk on the tree. In the real case the random walk is replaced by a drifted Brownian motion on the hyperbolic halfplane. In both cases the random processes are invariant under the automorphisms of the tree and the hyperbolic half-plane, respectively, that fix the point at infinity.

These random processes determine hitting distributions on the horocycles which, in a sense to be specified, are shown to be in the domain of attraction of $\alpha$-stable symmetric random variables. In both cases the exponent of $\alpha$-stability is related by an explicit formula to the drift coefficient.

\section{Introduction.}

There are many equivalent definitions of a stable symmetric random variable with values in the real field (for detailed treatments see $[\mathbf{G K}]$, or the more recent $[\mathbf{S T}]$ ). That which most easily extends to local fields is based on the form of its characteristic function: 
Definition 1.1. A symmetric random variable $U$ is stable if for some $\alpha>0$ and $c>0$ its characteristic function has the form

$$
\mathbf{E}\left[e^{i \xi U}\right]=e^{-c|\xi|^{\alpha}} \quad \text { for all } \xi .
$$

The constant $\alpha$, as opposed to the scaling factor $c$, plays an important role in the classification and properties of a stable random variable and the related stable processes, to the extent that one often refers to this as an $\alpha$-stable random variable. This constant is subject to the limitation $\alpha \leq 2$ : Indeed, if $\alpha>2$ then the second derivative of $e^{-c|\xi|^{\alpha}}$ must vanish at the origin, whence $\mathbf{E}\left[U^{2}\right]=0$.

The definition above extends readily to a local field, that is to a nondiscrete, totally disconnected, locally compact field. A local field $\mathcal{F}$ is endowed with a norm taking values in the non-negative reals and satisfying the ultrametric inequality:

$$
|a+b| \leq \max (|a|,|b|) .
$$

The norm is consequently subadditive, and $\mathcal{F}$ becomes a metric space with the induced distance. Local compactness implies completeness of $\mathcal{F}$ as a metric space. The norm is also multiplicative:

$$
|a b|=|a||b|,
$$

and the elements of unit norm form a compact group under multiplication. A random variable $U$ with values in $\mathcal{F}$ is symmetric if it has the same probability distribution as $a U$ for every $a \in \mathcal{F}$ with $|a|=1$.

The field $\mathcal{F}$ is a locally compact additive Abelian group, therefore we may associate to a random variable with values in $\mathcal{F}$ a characteristic function, defined as the (inverse) Fourier transform of its probability distribution. The character group $\hat{\mathcal{F}}$ of $\mathcal{F}$ is isomorphic to $\mathcal{F}$ itself (cf. $\S 2)$. Thus to every element $\xi \in \mathcal{F}$ we can associate a character $\chi_{\xi}$. The characteristic function of the random variable $U$ may then be defined as $\xi \mapsto \mathbf{E}\left[\chi_{\xi}(U)\right]$. At this point it is natural to define an $\alpha$-stable symmetric random variable to be a random variable $U$ with values in $\mathcal{F}$ whose characteristic function is of the type

$$
\mathbf{E}\left[\chi_{\xi}(U)\right]=e^{-c|\xi|^{\alpha}} \quad \text { for all } \xi \in \mathcal{F},
$$

for some $c>0$ and some $\alpha>0$ (cf. $[\mathbf{I}],[\mathbf{K}]$ ). This formal definition does not give any clue on the possible role of stable random variables in the definition of diffusion processes, neither does it immediately account for the role of the "geometry" of $\mathcal{F}$ with respect to stability.

In this paper we shall define, for each $\alpha>0$, an $\alpha$-stable symmetric random variable as the limit of sums of identically distributed independent random variables obtained as "hitting distributions" on a horocycle of a random walk on the Bruhat-Tits tree of $\mathcal{F}$. The number $\alpha$ arises in the definition of the random walk, measuring its drift; the isotropic (drift-free) 
random walk corresponds to the case $\alpha=1$. In other words, we "derive" a stable random variable via a construction which should have intuitive content, and should clarify the role of the scaling constant $c$ of (1.2). Even though multiplication of $U$ by a positive real number does not make sense in terms of the field operations, we shall be able to define, for an $\alpha$-stable symmetric random variable $U$ and for each $t>0$, an $\alpha$-stable symmetric random variable $U_{t}$ such that

$$
\mathbf{E}\left[\chi_{\xi}\left(U_{t}\right)\right]=\mathbf{E}\left[\chi_{\xi}(U)\right]^{t},
$$

as if $U_{t}$ were obtained multiplying $U$ by $t^{1 / \alpha}$. Thus we can define an $\alpha$-stable process on $\mathcal{F}$ with respect to real time (see also $[\mathbf{V}]$ ).

The relationship between the parameter $\alpha$ and the random walk used to derive $U$ should also clarify the role of the geometry of the field and its Bruhat-Tits tree in the definition of $\alpha$-stability. In fact the intuitive meaning of the drifted random walk on the tree may be translated in terms of the diffusion process $U_{t}$ : The larger is $\alpha$, the more probably we move into a smaller ball rather than out to a larger one. (The vertices of the BruhatTits tree are the balls of $\mathcal{F}$, see $\S 2$.) This intuitive content is elaborated in $[\mathbf{F 1}]$ in the context of finite ultrametric spaces, with compact ultrametric spaces as the limiting case.

It also turns out that the limitation $\alpha \leq 2$ is no longer in effect on a local field. This may actually be observed directly by just taking the Fourier transform of the function $e^{-c|\xi|^{\alpha}}$, and verifying that it is positive and continuous on $\mathcal{F}$ for every $\alpha>0[\mathbf{I}]$. Our derivation of the stable random variable also makes it clear that the limiting case is not $\alpha=2$, as in the real case, but rather $\alpha=\infty$, which corresponds to a bounded random variable with characteristic function identically 1 on a compact open subgroup of $\mathcal{F}$. In some respect this should be considered the analogue of a Gaussian random variable in this context $[\mathbf{E}]$.

In the second part of the paper we study a similar continuous-time situation. The role of the Bruhat-Tits tree of a local field is now played by the hyperbolic upper half-plane, and that of the random walk by a drifted Brownian motion, invariant under the hyperbolic isometries that fix the point at infinity; the horocycles through that point are the horizontal lines. The results obtained are remarkably similar. As it turns out, the drift-free random walk on the tree is easily replicated for a real-valued symmetric random variable with Cauchy distribution (corresponding to $\alpha=1$ ). In this case one only has to observe, using a time change, that the Cauchy distribution is the hitting distribution on the real line of a Brownian motion on the upper half-plane starting at $(0,1)$. For a drifted Brownian motion with downward drift coefficient $(\alpha-1) y / 2$, we obtain a hitting distribution which is in the domain of attraction of an $\alpha$-stable symmetric random variable. A 
drift coefficient strictly greater than $1 / 2$ always yields a hitting distribution with a finite second moment, that is in the domain of attraction of a Gaussian law. In the limiting case $\alpha=2$ the hitting distribution has infinite variance, but is in the extended attraction domain of a Gaussian law. These results are closely linked with results on exponential functionals of Brownian motions first obtained by Dufresne [D], motivated by applications to risk theory. We give a short alternate proof of Dufresne's result in the context of the hyperbolic half-plane.

The authors are aware of the fact that the results on random variables on local fields contained in this paper could be formulated in the more general context of locally compact ultrametric spaces, along the lines of [F1]. Indeed the algebraic structure of the field is blurred by the condition that the random variables be symmetric. The most general context for which our results and actual calculations would hold is that of locally compact, noncompact ultrametric spaces with the property that closed balls are compact and that the group of isometries acts transitively on the space. We chose to express and prove the result in the context of local fields mainly to stress the analogy with the theory of real-valued stable random variables.

Many ideas and actual computations for the first part of the paper were already explicit in $[\mathbf{F 1}]$ and [F2]. The third-named author presented a preliminary version of this part at a Conference commemorating Carl Herz in Montreal, and regrets that a delay in the preparation of the final version prevented the paper to be published in $[\mathbf{D M}]$; he would like to dedicate the paper to the memory of C. Herz.

Random variables and random processes in vector spaces with respect to local fields were studied extensively by S. Evans with quite different approach, methods and results. However, in the one-dimensional case his definition of Gaussian random variable $[\mathbf{E}]$ yields what we obtain in the limiting case $\alpha=\infty$, a random variable uniformly distributed on a compact open subgroup.

The authors acknowledge with thanks several helpful and illuminating comments by Tim Steger and Marc Yor.

\section{Notation and preliminaries.}

For the purpose of establishing notation we recall here the basic properties of local fields. The terminology is mostly the same as in [F3], to which we refer the reader for a more exhaustive description of the subject matter. Good general references on the subject are [T, Chapter 1], [W, Chapter 1]. Let $\mathcal{F}$ be a fixed local field. As explained above, its norm $|\cdot|$ satisfies the ultrametric inequality (1.1). Let $\mathcal{O}=\{a \in \mathcal{F}:|a| \leq 1\}$ be the compact and open ring of integers, $\mathcal{P}$ its maximal ideal, and $q$ the order of the finite field $\mathcal{O} / \mathcal{P}$. Fix a generator $\mathfrak{p}$ of $\mathcal{P}$. Then $|\mathfrak{p}|=1 / q$, and the norm in $\mathcal{F}$ 
takes exactly the values $q^{h}$ for $h \in \mathbf{Z}$, as well as the value 0 . Indeed any ball (necessarily open and closed) containing $a \in \mathcal{F}$ is of the form $a+\mathfrak{p}^{k} \mathcal{O}$ for some $k \in \mathbf{Z}$, and has radius $q^{-k}$ (we agree to refer to the radius of the "closed" ball, which is also its diameter, i.e., the maximum distance between any two of its points). Finally, denote by $\mathcal{O}^{\times}$the set of elements of unit norm, which is a (compact and open) group under multiplication.

A simple but very useful fact about the metric of $\mathcal{F}$ is that the set of balls can be identified in a natural fashion with (the set of vertices of) a homogeneous tree $\mathfrak{X}$ of degree $q+1$, often called the Bruhat-Tits tree associated to $\mathcal{F}$. A closed ball of radius (or diameter) $q^{h}$ is contained in exactly one ball of radius $q^{h+1}$ and contains exactly $q$ balls of radius $q^{h-1}$. So it is natural to let the balls be the vertices of a tree $\mathfrak{X}$, stipulating that two balls are adjacent if one is a maximal ball properly contained in the other. The boundary of $\mathfrak{X}$ may then be identified with the one-point compactification of $\mathcal{F}$, or better still with the projective line of $\mathcal{F}$. We shall let $\mathfrak{X}$ also stand for the set of vertices of the tree: Thus $x \in \mathfrak{X}$ means that $x$ is a closed ball of the metric space $\mathcal{F}$.

An important role in our construction will be played by the so-called horocycles of the tree with respect to the point at infinity $\omega$, which is the boundary point corresponding to the infinite chain of vertices $\left\{\mathfrak{p}^{-n} \mathcal{O}: n=\right.$ $0,1, \ldots\}$. Each horocycle consists of balls of the same radius, which constitute a partition of $\mathcal{F}$.

The Haar measure $m$ on $\mathcal{F}$ may be chosen in such a way that $m(\mathcal{O})=1$. Once this choice is made, the measure of each ball equals its diameter. The Haar integral of, say, a continuous function $u$ is denoted by $\int_{\mathcal{F}} u(a) d a$, or simply by $\int_{\mathcal{F}} u$. With our definitions the substitution $a=\mathfrak{p}^{n} b$ yields

$$
d b=q^{n} d a
$$

a simple fact which will be exploited in the sequel.

We describe now the character group of $\mathcal{F}$. Observe first that every character maps $\mathcal{O}$ into a compact subgroup of the 1-dimensional torus. It follows that the image of $\mathcal{O}$ is finite, hence the kernel of every non-trivial character is open and compact. This means that we can find a character $\chi$ whose kernel is $\mathcal{O}$. (If the kernel of $\chi^{\prime}$ is $\mathfrak{p}^{k} \mathcal{O}$ then let $\chi(a)=\chi^{\prime}\left(\mathfrak{p}^{-k} a\right)$ for every $a \in \mathcal{F}$.) Fix such a $\chi$. For each $\xi \in \mathcal{F}$ define a character $\chi_{\xi}$ by $\chi_{\xi}(a)=\chi(\xi a)$. These characters $\chi_{\xi}$ form a group under multiplication which is isomorphic to $\mathcal{F}$. On the other hand, if $f \in L^{1}(\mathcal{F})$ and $\hat{f}(\xi)=\int_{\mathcal{F}} f \chi_{\xi}=0$ for every $\xi \in \mathcal{F}$, then $f=0$. Thus the full character group of $\mathcal{F}$ may be identified with $\mathcal{F}$ itself via the correspondence $\chi_{\xi} \mapsto \xi$. 
For the computations of this paper we make use of the so-called spherical functions. First set

$$
\phi_{0}(a)= \begin{cases}1 & \text { if }|a| \leq 1, \\ \frac{1}{1-q} & \text { if }|a|=q, \\ 0 & \text { otherwise }\end{cases}
$$

then, for $n \in \mathbf{Z}$, define $\phi_{n}$ by

$$
\phi_{n}(a)=\phi_{0}\left(\mathfrak{p}^{-n} a\right)
$$

finally set

$$
\phi_{-\infty} \equiv 1 \text {. }
$$

The functions $\phi_{n}$, for $n \in \mathbf{Z} \cup\{-\infty\}$, are called the spherical functions of $\mathcal{F}$, with respect to its group of isometries $[$ F3]. The spherical functions other than $\phi_{-\infty}$ form an orthogonal system in $L^{2}(\mathcal{F})$ that spans the subspace of radial functions (i.e., constant on the sets $\left\{a \in \mathcal{F}:|a|=q^{h}\right\}$ for $h \in \mathbf{Z}$ ).

Spherical functions may be obtained as averages of characters. If $\chi_{\xi}$ is a character and $|\xi|=q^{n}$ then

$$
\phi_{n}(a)=\int_{\mathcal{O}^{\times}} \chi_{\xi}(u a) d \mu(u),
$$

where $d \mu$ denotes the normalized Haar measure on the group $\mathcal{O}^{\times}$. course the trivial spherical function is the average of, and coincides with, the trivial character.) This can be checked by direct computation or using one of the standard characterizations of spherical functions [L, Chap. 4], e.g., the fact that they yield multiplicative linear functionals on the commutative convolution algebra of radial integrable functions. The computation of the Fourier transform of a radial function therefore reduces to the much easier computation of its spherical transform.

The upper half-plane

$$
\Pi=\left\{z=(x, y) \in \mathbf{C}=\mathbf{R}^{2}: y>0\right\}
$$

is a homogeneous Riemannian manifold when endowed with the Riemannian metric $d s^{2}=|d z|^{2} / y^{2}$. This metric is conformal to the Euclidean metric, and the corresponding Laplace-Beltrami operator $\Delta_{\Pi}$ is a non-constant multiple of the Euclidean Laplacian, namely $\Delta_{\Pi}=y^{2} \Delta$. The group $G$ of orientation-preserving isometries of $\Pi$ is $S L(2, \mathbf{R}) / \pm 1$, acting as fractional linear transformations. Thus $\Pi=G / \mathbf{T}$ is a Riemannian symmetric space, where the one-dimensional torus $\mathbf{T}$ of rotation matrices is the isotropy subgroup at $z=i$ of $G$. The horocycles of $\Pi$, i.e., the orbits of the nilpotent subgroup $N$ of an Iwasawa decomposition of $G=K A N$, are all Euclidean circles tangent to the real line, as well as all horizontal lines; those of the latter type are exactly the horocycles passing through the point at infinity $\infty$. The orientation-preserving isometries that fix $\infty$, thereby preserving the 
family of such horizontal horocycles, are of the form $z \mapsto a z+b$, with $a>0$ and $b \in \mathbf{R}$. Among these, horizontal translations (i.e., those for $a=1$ ) preserve each horizontal horocycle. It follows that the induced metric on the horizontal line $\left\{y=y_{0}\right\}$ is the constant $1 / y_{0}$ times the Euclidean metric.

\section{The case of a local field.}

Our aim is to construct for each $\alpha>0$ a symmetric random variable $U$ with values in $\mathcal{F}$ and such that (1.2) holds with $c=1$. We shall first define a symmetric random variable $V$ uniformly distributed on each ball of radius 1 . Of course $V$ cannot be stable because its characteristic function vanishes on a non-negligible set of characters, namely $\left\{\chi_{\xi}:|\xi| \geq q\right\}$ (see below). However we shall eventually obtain any prescribed $\alpha$-stable random variable $U$ as a limit of appropriate multiples of sums of independent copies of $V$.

Consider the random walk on the tree $\mathfrak{X}$ of $\mathcal{F}$ given by the following transition matrix:

$$
P(x, y)= \begin{cases}0 & \text { if } x, y \text { are not adjacent } \\ \frac{1}{1+q^{\alpha}} & \text { if } x, y \text { are adjacent and } x \subset y, \\ \frac{1}{q}\left(1-\frac{1}{1+q^{\alpha}}\right) & \text { if } x, y \text { are adjacent and } y \subset x .\end{cases}
$$

The number $P(x, y)$ is interpreted of course as the probability of going from $x$ to $y$ in one step. The only case in which the random walk is symmetric is when $\alpha=1$, for which the transition matrix $P(x, y)$ induces the isotropic nearest-neighbor random walk on $\mathfrak{X}$. If $\alpha<1$ the probability of moving towards $\omega$ is greater than that of moving towards one of the points of $\mathcal{F}$; the opposite happens if $\alpha>1$. But in all cases $1 /\left(1+q^{\alpha}\right)<1 / 2$, thus with probability 1 the random walk eventually moves to smaller and smaller balls.

Define a positive function on the set of balls of radius 1 , that is, on the horocycle of the tree $\mathfrak{X}$ (with respect to $\omega$ ) containing the ball $\mathcal{O}$ : If $x=a+\mathcal{O}$ is a ball of radius 1 , we let $f(x)$ be the probability that $x$ is the first ball of radius 1 reached by the random walk if its starting point is the ball $\mathfrak{p}^{-1} \mathcal{O}$. The function $f$ is defined on the quotient $\mathcal{F} / \mathcal{O}$, thus it may be regarded as a function defined on $\mathcal{F}$ which is constant on balls of radius 1 . Then

$$
\int_{\mathcal{F}} f=\sum f(a+\mathcal{O})=1,
$$

the sum being taken over all the disjoint balls $a+\mathcal{O}$ of radius 1 , which, by our choice of the Haar measure, have all measure 1.

Let $V$ be the random variable with values in $\mathcal{F}$ which is uniformly distributed with probability $f(a)$ on each ball $a+\mathcal{O}$ of radius 1 . We proceed to compute the density $f$ of the probability distribution of $V$. Let $x_{0}, x_{1}, \ldots$ 
be the infinite chain on the tree which starts at $x_{0}=\mathcal{O}$ and approaches $\omega$; explicitly, $x_{j}=\mathfrak{p}^{-j} \mathcal{O}$ for $j=0,1, \ldots$ Let $\zeta_{j}$ be the probability of reaching sooner or later $x_{j+1}$ under the condition that $x_{j}$ is reached at least once. Since we start from $x_{1}$ we have $\zeta_{1}=1 /\left(1+q^{\alpha}\right)$. For $j>1$ we must have

$$
\zeta_{j}=\frac{1}{1+q^{\alpha}}+\left(1-\frac{1}{1+q^{\alpha}}\right) \zeta_{j-1} \zeta_{j}
$$

In other words, once the walk is at $x_{j}$ it can either reach $x_{j+1}$ with the next step, which happens with probability $1 /\left(1+q^{\alpha}\right)$, or else, with probability $1-1 /\left(1+q^{\alpha}\right)$, it will first move to one of the balls contained in $x_{j}$, then eventually reach again $x_{j}$ with probability $\zeta_{j-1}$, and from there eventually reach $x_{j+1}$ with probability $\zeta_{j}$. It follows that

$$
\zeta_{j}=\frac{q^{j \alpha}-1}{q^{(j+1) \alpha}-1} \quad \text { for } j \geq 1
$$

Observe that

$$
f\left(x_{0}\right)=\sum_{k=1}^{\infty} q^{-k}\left(1-\zeta_{k}\right) \prod_{j=1}^{k-1} \zeta_{j}
$$

(the empty product equals 1 ). Indeed the summands are the probabilities of the disjoint events $\Omega_{k}$ defined as follows: The random walk reaches, sooner or later, the vertex $x_{k}$ without ever reaching $x_{k+1}$, and, of the possible $q^{k}$ balls of radius 1 contained in the ball $x_{k}$, the first reached is $x_{0}$. This determines $f(a)$ for all $a \in \mathcal{O}=x_{0}$, but $f$ will obviously also take the same value on each of the $q-1$ balls of radius 1 contained in $\mathfrak{p}^{-1} \mathcal{O}$. At this point $f(a)$ is determined and given by the above sum for all $|a| \leq q$. To reach any other ball the random walk must necessarily reach $x_{2}$. More generally, in order to reach a ball of radius 1 of type $a+\mathcal{O}$ with $|a|=q^{n}$, the random walk must reach $x_{n}=\mathfrak{p}^{-n} \mathcal{O}$. Thus

$$
f(a)=\sum_{k=n}^{\infty} q^{-k}\left(1-\zeta_{k}\right) \prod_{j=1}^{k-1} \zeta_{j} \quad \text { where } n=\max \left\{1, \log _{q}|a|\right\} .
$$

Let us compute the spherical transform of $f$, i.e., the scalar products

$$
\left\langle f, \phi_{n}\right\rangle=\int_{\mathcal{F}} f \phi_{n} \quad \text { for } n \in \mathbf{Z} \cup\{-\infty\} .
$$

Recall that for $n \in \mathbf{Z}$ the spherical function $\phi_{n}$ is supported on the set $\mathfrak{p}^{n-1} \mathcal{O}=\left\{a \in \mathcal{F}:|a| \leq q^{-n+1}\right\}$, and its integral vanishes. But $f$ is constant on $\mathfrak{p}^{-1} \mathcal{O}$, therefore

$$
\left\langle f, \phi_{n}\right\rangle=0 \quad \text { for } n \geq 0 .
$$

To compute $\left\langle f, \phi_{-k}\right\rangle$ for $k \geq 1$ observe that $f$ is constant on each of the sets $\left\{a \in \mathcal{F}:|a|=q^{j}\right\}$. For $j>1$ this set has measure $q^{j}-q^{j-1}$, while 
$\{a \in \mathcal{F}:|a| \leq q\}$ has measure $q$. If $a_{1}, \ldots, a_{k+1} \in \mathcal{F}$ are such that $\left|a_{j}\right|=q^{j}$ for all $j=1, \ldots, k+1$, then

$$
\begin{aligned}
\left\langle f, \phi_{-k}\right\rangle & =q f\left(a_{1}\right)+\sum_{j=2}^{k}\left(q^{j}-q^{j-1}\right) f\left(a_{j}\right)+\frac{1}{1-q}\left(q^{k+1}-q^{k}\right) f\left(a_{k+1}\right) \\
& =\sum_{j=1}^{k} q^{j}\left(f\left(a_{j}\right)-f\left(a_{j+1}\right)\right)=\sum_{j=1}^{k}\left(1-\zeta_{j}\right) \prod_{i=1}^{j-1} \zeta_{i} \\
& =1-\prod_{i=1}^{k} \zeta_{i}=1-\frac{q^{\alpha}-1}{q^{(k+1) \alpha}-1}
\end{aligned}
$$

since $\left(q^{k+1}-q^{k}\right) /(1-q)=-q^{k}$ and $f\left(a_{j}\right)-f\left(a_{j+1}\right)=q^{-j}\left(1-\zeta_{j}\right) \prod_{i=1}^{j-1} \zeta_{i}$, from (3.3), and using (3.2).

Recall that $V$ is the random variable that on each ball of radius 1 , of the type $a+\mathcal{O}$, is uniformly distributed with probability $f(a)$. Clearly if $a \in \mathcal{F}$ with $|a|=1$ the random variables $a V$ and $V$ have the same probability distribution. Observe that the density of the probability distribution of $\mathfrak{p}^{n} V$ is $f_{n}(a)=q^{n} f\left(\mathfrak{p}^{-n} a\right)$. Therefore, for $n>k$,

$$
\begin{aligned}
\left\langle f_{n}, \phi_{n-k}\right\rangle & =q^{n} \int_{\mathcal{F}} f\left(\mathfrak{p}^{-n} a\right) \phi_{n-k}(a) d a=q^{n} \int_{\mathcal{F}} f\left(\mathfrak{p}^{-n} a\right) \phi_{-k}\left(\mathfrak{p}^{-n} a\right) d a \\
& =\int_{\mathcal{F}} f(b) \phi_{-k}(b) d b=1-\frac{q^{\alpha}-1}{q^{(k+1) \alpha}-1},
\end{aligned}
$$

having changed variable $a=\mathfrak{p}^{n} b$ and used (2.1). Thus, for $h \in \mathbf{Z}$ and $n>h$, setting $h=n-k$ one has:

$$
\left\langle f_{n}, \phi_{h}\right\rangle=1-\frac{q^{\alpha}-1}{q^{(n+1) \alpha} q^{-h \alpha}-1} .
$$

Let $V_{1}, V_{2}, \ldots$ be a sequence of independent random variables with the same distribution as $V$, and let

$$
U_{n}=\mathfrak{p}^{n} \sum_{k=1}^{j(n)} V_{k} \quad \text { for } n \geq 1,
$$

where, denoting by $\lfloor t\rfloor$ the integral part of the real number $t$, we set

$$
j(n)=\left\lfloor\frac{q^{(n+1) \alpha}}{q^{\alpha}-1}\right\rfloor .
$$

The density of the probability distribution of $U_{n}$ is simply the $j(n)$-fold convolution product $F_{n}=f_{n} * \cdots * f_{n}$, hence (3.4) gives

$$
\left\langle F_{n}, \phi_{h}\right\rangle=\left(1-\frac{q^{\alpha}-1}{q^{(n+1) \alpha} q^{-h \alpha}-1}\right)^{j(n)} .
$$


For fixed $h$ we obtain in the limit

$$
\lim _{n \rightarrow \infty}\left\langle F_{n}, \phi_{h}\right\rangle=e^{-q^{h \alpha}} .
$$

Define the random variable $U$ as a limit in distribution of $U_{n}$. More precisely, keeping in mind that $\left\{\phi_{n}: n \in \mathbf{Z}\right\}$ is an orthogonal set in $L^{2}(\mathcal{F})$ whose span is the space of radial functions, and recalling that the square of the $L^{2}$ norm of $\phi_{k}$ is $q^{-k} q /(q-1)$, define the radial function $F$ on $\mathcal{F}$ as

$$
F(a)=\sum_{k=-\infty}^{+\infty}\left\|\phi_{k}\right\|^{-2} e^{-q^{k \alpha}} \phi_{k}(a)=\frac{q-1}{q} \sum_{k=-\infty}^{+\infty} q^{k} e^{-q^{k \alpha}} \phi_{k}(a) .
$$

Then on each radial set $\left\{a \in \mathcal{F}:|a|=q^{j}\right\}$ we have $F=\lim _{n} F_{n}$ uniformly. Let $U$ be the random variable whose probability distribution has density $F$. The characteristic function of $U$ simply coincides with the Fourier transform of $F$ given by

$$
\hat{F}(\xi)=\int_{\mathcal{F}} F \chi_{\xi}=\int_{\mathcal{F}} F \phi_{h}=e^{-q^{h \alpha}} \quad \text { for }|\xi|=q^{h} .
$$

We have thus proved:

Theorem 3.1. The random variable $U$ defined above has characteristic function

$$
\mathbf{E}\left[\chi_{\xi}(U)\right]=e^{-|\xi|^{\alpha}} \quad \text { for } \xi \in \mathcal{F} .
$$

In other words, $U$ is a symmetric $\alpha$-stable random variable in "standard form" (i.e., with scaling factor $c=1$ ).

To clarify the role of the scaling factor, set

$$
j(n, t)=\left\lfloor\frac{t q^{(n+1) \alpha}}{q^{\alpha}-1}\right\rfloor \quad \text { for } n \geq 1 \text { and real } t>0 .
$$

Then the random variables

$$
U_{n, t}=\mathfrak{p}^{n} \sum_{k=1}^{j(n, t)} V_{k}
$$

converge in distribution to a random variable $U_{t}$ with characteristic function

$$
\mathbf{E}\left[\chi_{\xi}\left(U_{t}\right)\right]=e^{-t|\xi|^{\alpha}} \quad \text { for } \xi \in \mathcal{F} .
$$

The density $F_{t}$ of the probability distribution of $U_{t}$ satisfies $F_{t+s}=F_{t} * F_{s}$ whenever $s, t>0$, and may be interpreted as the kernel of a diffusion process $[\mathbf{F} 1],[\mathbf{V}]$.

The stable random variable $U$ has the property that, if $U_{(1)}, U_{(2)}, \ldots$ are independent random variables with the same distribution as $U$, defining 
$\tilde{\jmath}(n)=\left\lfloor q^{n \alpha}\right\rfloor$ then

$$
\mathfrak{p}^{n} \sum_{k=1}^{\tilde{\jmath}(n)} U_{(k)}
$$

converges in distribution to $U$. To prove this, observe that the density of the probability distribution of $\mathfrak{p}^{n} U$ is $H_{n}(a)=q^{n} F\left(\mathfrak{p}^{-n} a\right)$, and that, for $n$ large and $n-k=h$,

$$
\left\langle H_{n}, \phi_{h}\right\rangle=q^{n} \int_{\mathcal{F}} F\left(\mathfrak{p}^{-n} a\right) \phi_{-k}\left(\mathfrak{p}^{-n} a\right) d a=\int_{\mathcal{F}} F \phi_{-k}=e^{-q^{(h-n) \alpha}} .
$$

Therefore if $G_{n}=H_{n} * \cdots * H_{n}$ (with $\tilde{\jmath}(n)$ factors) then

$$
\left\langle G_{n}, \phi_{h}\right\rangle=e^{-\tilde{\jmath}(n) q^{(h-n) \alpha}} .
$$

The definition of $\tilde{\jmath}(n)$ implies that

$$
\lim _{n \rightarrow \infty}\left\langle G_{n}, \phi_{h}\right\rangle=e^{-q^{h \alpha}},
$$

which proves our assertion.

\section{The hyperbolic half-plane.}

The real line plays with reference to the hyperbolic half-plane $\Pi$ the same role as the local field $\mathcal{F}$ plays with reference to the homogeneous tree $\mathfrak{X}$ of degree $q+1$. In other words the boundary of $\Pi$ can be realized as the projective line $\mathbf{R} \cup\{\infty\}$. The role of a drifted random walk on $\mathfrak{X}$ is played in this context by a random process defined by

$$
\left\{\begin{array}{l}
d X_{t}=Y_{t} d W_{t}^{(1)}, \\
d Y_{t}=Y_{t} d W_{t}^{(2)}-\left(\nu-\frac{1}{2}\right) Y_{t} d t,
\end{array}\right.
$$

where $W_{t}^{(1)}, W_{t}^{(2)}$ are independent one-dimensional Brownian motions. The real coefficient $\nu-1 / 2$ measures the strength of the drift (positive for downward drift, negative for upward). Observe that the process is invariant under the orientation-preserving isometries of $\Pi$ that fix the point at infinity, that is, under the real affine transformations $z \mapsto a z+b$ with $a>0$ and $b \in \mathbf{R}$. The infinitesimal generator that corresponds to (4.1) is

$$
L=\frac{y^{2}}{2} \Delta-\left(\nu-\frac{1}{2}\right) y \frac{\partial}{\partial y} .
$$

Of course $L$ is also invariant under the same affine transformations.

For $a>0$ let $H_{a}$ be the horocycle through $i a=(0, a)$ and through the point at infinity; thus $H_{a}$ is the horizontal line given by $\{\Im z=a\}$. Consider the process starting at the point $i=(0,1)$, above the $x$-axis. For $0<a<1$ let $\tau_{a}$ denote the hitting time on $H_{a}$, and let $\Xi^{a}=X_{\tau_{a}}$ be the corresponding hitting random variable. 
Let us compute the characteristic function of $\Xi^{a}$. For $y>0$, the solution of $(4.1)$ with starting point $i y=(0, y)$ is

$$
\begin{aligned}
Y_{y, t} & =y e^{W_{t}^{(2)}-\nu t}, \\
X_{y, t} & =\int_{0}^{t} Y_{y, s} d W_{s}^{(1)} .
\end{aligned}
$$

For $\nu>0$ and every $y>0$ this implies that $Y_{y, t} \rightarrow 0$ almost surely as $t \rightarrow \infty$, so that $\tau_{a}<\infty$ almost surely. Let us first compute the characteristic function of $X_{y, \infty}$. Introducing the random variable

$$
A_{t}^{\nu}=\frac{1}{y^{2}} \int_{0}^{t} Y_{y, s}^{2} d s=\int_{0}^{t} e^{2 W_{s}^{(2)}-2 \nu s} d s
$$

observe that

$$
X_{y, t} \text { has the same law as } \gamma\left(\int_{0}^{t} Y_{y, s}^{2} d s\right)=\gamma\left(y^{2} A_{t}^{\nu}\right),
$$

where $\gamma(t)$ is a Brownian motion independent of $W_{t}^{(1)}, W_{t}^{(2)}$. Thus $X_{y, \infty}$ has the same law as $\gamma\left(y^{2} A_{\infty}^{\nu}\right)$. By a result of Dufresne [D] (see also Yor $[\mathbf{Y}]$ ), the random variable $A_{\infty}^{\nu}$ has the same law as the reciprocal of $2 Z$, where $Z$ is a random variable having law $\Gamma(\nu, 1)$. That is, $Z$ has a density

$$
g(z)= \begin{cases}\frac{z^{\nu-1} e^{-z}}{\Gamma(\nu)} & \text { for } z>0 \\ 0 & \text { for } z \leq 0\end{cases}
$$

The Laplace transform of $A_{\infty}^{\nu}$ for $t>0$ is

$$
\psi(t)=\mathbf{E}\left[e^{-t A_{\infty}^{\nu}}\right]=\mathbf{E}\left[e^{-t /(2 Z)}\right]=\frac{1}{\Gamma(\nu)} \int_{0}^{\infty} e^{-t /(2 z)} z^{\nu-1} e^{-z} d z .
$$

Since the modified Bessel function of order $\nu$ [GR, $\S \S 8.4-8.5]$ is given by [GR, Formula 8.432.6]

$$
K_{\nu}(z)=\frac{2^{\nu-1}}{z^{\nu}} \int_{0}^{\infty} t^{\nu-1} e^{-\left(z^{2} / 4 t+t\right)} d t
$$

then, setting for later convenience

$$
\tilde{K}_{\nu}(z)=\frac{2^{1-\nu}}{\Gamma(\nu)}|z|^{\nu} K_{\nu}(|z|),
$$

we have

$$
\psi\left(z^{2} / 2\right)=\tilde{K}_{\nu}(z) .
$$

Using the independence of the Brownian motion $\gamma(t)$ and the random variable $A_{\infty}^{\nu}$, one has easily

$$
\mathbf{E}\left[e^{i \lambda X_{y, \infty}}\right]=\mathbf{E}\left[e^{i \lambda \gamma\left(y^{2} A_{\infty}^{\nu}\right)}\right]=\mathbf{E}\left[e^{-\left(\lambda^{2} / 2\right) y^{2} A_{\infty}^{\nu}}\right]=\tilde{K}_{\nu}(\lambda y) .
$$


Remark 4.1. Recall that the characteristic function of a Student distribution $t(n)$ with $n$ degrees of freedom is

$$
\sigma_{n}(\lambda)=\tilde{K}_{n / 2}(|\lambda| \sqrt{n}) .
$$

Comparing with (4.6) for $y=1$ we find again that, if $\nu=1 / 2$, then $X_{1, \infty}$ has a Cauchy distribution (which is the same as a $t(1)$ ). Whereas, if $\nu=n / 2$ for $n \geq 2$, then $X_{1, \infty}$ has the same distribution as a $t(n)$ divided by $\sqrt{n}$.

Since the density of $\left(2 A_{\infty}^{\nu}\right)^{-1}$ is given by (4.3), then that of $X_{1, \infty}$ is

$$
\begin{aligned}
f(x) & =\mathbf{E}\left[\frac{1}{\sqrt{2 \pi A_{\infty}^{\nu}}} e^{-x^{2} /\left(2 A_{\infty}^{\nu}\right)}\right]=\frac{1}{\Gamma(\nu) \sqrt{\pi}} \int_{0}^{\infty} s^{(\nu+1 / 2)-1} e^{-s x^{2}} e^{-s} d s \\
& =\frac{\Gamma(\nu+1 / 2)}{\Gamma(\nu) \sqrt{\pi}} \frac{1}{\left(1+x^{2}\right)^{\nu+1 / 2}}
\end{aligned}
$$

(cf. [AG, last formula of the paper] for $\nu$ a half-integer).

By (4.2) the characteristic function $\phi_{a}(\lambda)$ of $\Xi^{a}=X_{1, \tau_{a}}$ is the same as that of $\gamma\left(A_{\tau_{a}}^{\nu}\right)$. With reference to (4.6) for $y=1$, we have

$$
e^{-\left(\lambda^{2} / 2\right) A_{\infty}^{\nu}}=e^{-\left(\lambda^{2} / 2\right) \int_{0}^{\tau_{a}} Y_{1, s}^{2} d s} e^{-\left(\lambda^{2} / 2\right) \int_{\tau_{a}}^{\infty} Y_{1, s}^{2} d s} .
$$

The strong Markov property then implies

$$
\mathbf{E}\left[e^{i \lambda X_{1, \infty}}\right]=\mathbf{E}\left[e^{-\left(\lambda^{2} / 2\right) A_{\infty}^{\nu}}\right]=\mathbf{E}\left[e^{-\left(\lambda^{2} / 2\right) \int_{0}^{\tau_{a}} Y_{1, s}^{2} d s}\right] \mathbf{E}\left[e^{-\left(\lambda^{2} / 2\right) \int_{0}^{\infty} Y_{a, s}^{2} d s}\right],
$$

whose rightmost factor equals $\mathbf{E}\left[e^{i \lambda X_{a, \infty}}\right]$. Then, by (4.6),

$$
\tilde{K}_{\nu}(\lambda)=\phi_{a}(\lambda) \tilde{K}_{\nu}(\lambda a)
$$

whence

$$
\phi_{a}(\lambda)=\mathbf{E}\left[e^{i \lambda X_{1, \tau_{a}}}\right]=\frac{\tilde{K}_{\nu}(\lambda)}{\tilde{K}_{\nu}(\lambda a)} .
$$

Remark 4.2. An easy proof of Dufresne's result [D] used above can be obtained using the diffusion $Y$ defined in (4.1). Indeed, for fixed $\lambda$, it is easy to check that the function $u(y)=\tilde{K}_{\nu}(\lambda y)$ is a positive solution of the problem

$$
\left\{\begin{array}{l}
\frac{1}{2} y^{2} u^{\prime \prime}(y)-\left(\nu-\frac{1}{2}\right) y u^{\prime}(y)-\frac{\lambda^{2}}{2} y^{2} u(y)=0 \quad \text { for } y>0 \\
u(0)=1 .
\end{array}\right.
$$

This is straightforward, using the defining differential equation of the modified Bessel function $K_{\nu}$, namely

$$
z^{2} K_{\nu}^{\prime \prime}(z)+z K_{\nu}^{\prime}(z)-\left(z^{2}+\nu^{2}\right) K_{\nu}(z)=0 .
$$


Moreover $u(y) \rightarrow 0$ as $y \rightarrow \infty$. Using this and the fact that $\lim _{t \rightarrow \infty} Y_{y, t}=0$ almost surely, it is easy to prove the Feynman-Kac formula

$$
u(y)=\mathbf{E}\left[e^{-\left(\lambda^{2} / 2\right) \int_{0}^{\infty} Y_{y, s}^{2} d s}\right]=\mathbf{E}\left[e^{-\left(\lambda^{2} / 2\right) y^{2} A_{\infty}^{\nu}}\right] .
$$

Theorem 4.3. If $0<\nu \leq 1$ then, for every $0 \leq a<1$, the random variable $\Xi^{a}$ lies in the domain of attraction of a symmetric $\alpha$-stable real-valued random variable, where $\alpha=2 \nu$. In the special case $\nu=1 / 2($ i.e., $\alpha=1)$, corresponding to drift-free Brownian motion, the random variable $\Xi^{a}$ is itself distributed according the Cauchy law.

The limiting case $\nu=1$ (i.e., $\alpha=2$ ) yields a random variable $\Xi^{a}$ which is in the extended domain of attraction of a Gaussian random variable.

For $\nu>1$, the random variable $\Xi^{a}$, which has a moment of order 2 , is in the domain of attraction of a Gaussian random variable.

Proof. We shall give the details for $a>0$, the changes for the case $a=0$ being almost obvious.

Consider first the case $0<\nu<1$. In order to prove that $\Xi^{a}=X_{\tau_{a}}$ is in the domain of attraction of an $\alpha$-stable random variable, with $\alpha=2 \nu$, it suffices to prove that for some $c>0$ we have

$$
\lim _{n \rightarrow \infty} \phi_{a}\left(\frac{\theta}{n^{1 / \alpha}}\right)^{n}=e^{-c|\theta|^{\alpha}}
$$

that is,

$$
\lim _{n \rightarrow \infty} n\left(1-\phi_{a}\left(\frac{\theta}{n^{1 / \alpha}}\right)\right)=c|\theta|^{\alpha} .
$$

To this end observe that

$$
1-\phi_{a}(\lambda)=1-\frac{\tilde{K}_{\nu}(\lambda)}{\tilde{K}_{\nu}(\lambda a)}=\frac{\tilde{K}_{\nu}(\lambda a)-\tilde{K}_{\nu}(\lambda)}{\tilde{K}_{\nu}(\lambda a)} .
$$

Recalling (4.4), (4.5) one has

$$
\lim _{z \rightarrow 0^{+}} \tilde{K}_{\nu}(z)=1 .
$$

It remains to prove that

$$
\lim _{n \rightarrow \infty} n\left(\tilde{K}_{\nu}\left(\frac{a \theta}{n^{1 / \alpha}}\right)-\tilde{K}_{\nu}\left(\frac{\theta}{n^{1 / \alpha}}\right)\right)=c|\theta|^{\alpha} .
$$

In fact

$$
\begin{aligned}
n\left(\tilde{K}_{\nu}\left(\frac{a \theta}{n^{1 / \alpha}}\right)\right. & \left.-\tilde{K}_{\nu}\left(\frac{\theta}{n^{1 / \alpha}}\right)\right) \\
& =\frac{n}{\Gamma(\nu)} \int_{0}^{\infty} t^{\nu-1} e^{-t}\left(e^{-a^{2} \theta^{2} / 4 n^{2 / \alpha} t}-e^{-\theta^{2} / 4 n^{2 / \alpha} t}\right) d t \\
& =\frac{1}{\Gamma(\nu)} \int_{0}^{\infty} s^{\nu-1} e^{-s n^{-2 / \alpha}}\left(e^{-a^{2} \theta^{2} / 4 s}-e^{-\theta^{2} / 4 s}\right) d s
\end{aligned}
$$


(the last factor of the integrand is positive for $0<a<1$ ). For $n \rightarrow \infty$ we obtain

$\frac{1}{\Gamma(\nu)} \int_{0}^{\infty} s^{\nu-1}\left(e^{-a^{2} \theta^{2} / 4 s}-e^{-\theta^{2} / 4 s}\right) d s=\frac{|\theta|^{2 \nu}}{\Gamma(\nu)} \int_{0}^{\infty} u^{\nu-1}\left(e^{-a^{2} / 4 u}-e^{-1 / 4 u}\right) d u$.

This integral is always convergent because the factor in parentheses is asymptotic to $c / u$ as $u \rightarrow \infty$.

The case $\nu>1$ is immediate, since $X_{1, \infty}$ has a finite second moment by the expression of its density given in (4.7). Therefore $\tilde{K}_{\nu}$ is differentiable twice by (4.6), then so is $\phi_{a}$ by (4.8), thus ensuring that $\Xi^{a}$ also has a finite second moment.

Finally, in the case $\nu=1$ it is sufficient to show that for some sequence $b_{n} \rightarrow \infty$ we have

$$
\lim _{n \rightarrow \infty} n\left(1-\phi_{a}\left(\frac{\theta}{b_{n}}\right)\right)=c \theta^{2} .
$$

As in the case $0<\nu<1$, this is the same as proving that

$$
\lim _{n \rightarrow \infty} n\left(\tilde{K}_{1}\left(\frac{a \theta}{b_{n}}\right)-\tilde{K}_{1}\left(\frac{\theta}{b_{n}}\right)\right)=c \theta^{2} .
$$

We have

$$
\tilde{K}_{1}\left(\frac{a \theta}{b_{n}}\right)-\tilde{K}_{1}\left(\frac{\theta}{b_{n}}\right)=\int_{a \theta / b_{n}}^{\theta / b_{n}} t K_{0}(t) d t
$$

since $d \tilde{K}_{1}(z) / d z=-z K_{0}(z)$ by [GR, Formula 8.486.14]. By [AS, Formula 9.6.8] the function $K_{0}(z)$ is asymptotic to $-\log z$ for $z \rightarrow 0^{+}$, therefore we want to evaluate the limit for $n \rightarrow \infty$ of

$$
-n \int_{a \theta / b_{n}}^{\theta / b_{n}} t \log t d t=\left.\frac{n t^{2}(1-2 \log t)}{4}\right|_{a \theta / b_{n}} ^{\theta / b_{n}},
$$

which is finite and positive if $b_{n}=\sqrt{n \log n}$.

We are in a position to replicate on the real line, for $0<\alpha<2$, the procedure we followed for local fields in the preceding section. Fix $0<a<1$ (e.g., $a=1 / 2$ ), and let $V_{1}, V_{2}, \ldots$ be a sequence of independent random variables with the same distribution as $\Xi^{a}$. By Theorem 4.3, the sequence

$$
U_{n}=n^{-1 / \alpha} \sum_{k=1}^{n} V_{k}
$$

converges in law to a symmetric $\alpha$-stable random variable $U$ on the real line.

Remark 4.4. As indicated in the statement of Theorem 4.3, the hitting distribution on a horocycle $H_{a}$ or on the boundary $\mathbf{R}$ of a drift-free Brownian motion on $\Pi$ is the Cauchy distribution, up to a scaling factor. The situation is completely different for local fields, where the hitting distributions of an isotropic random walk on any horocycle as well as on the boundary $\mathcal{F}$ 
itself are constant on a compact open subgroup. This implies that their characteristic functions have compact support, and cannot be of the type $e^{-c|\xi|}$. In our terminology they are not distributed according a Cauchy law.

We remark however that the hitting distribution on the boundary of a tree of an isotropic random walk is called a Cauchy distribution in $[\mathbf{H}]$.

\section{References}

[AS] M. Abramowitz and I.A. Stegun (eds.), Handbook of Mathematical Functions with Formulas, Graphs, and Mathematical Tables, Dover, New York, 1992; MR 94b:00012.

[AG] L. Alili and J.-C. Gruet, An explanation of a generalized Bougerol's identity in terms of hyperbolic Brownian motion, Exponential Functionals and Principal Values Related to Brownian Motion (M. Yor, ed.), Bibl. Rev. Mat. Iberoamericana, Rev. Mat. Iberoam., Madrid, (1997), 15-33; MR 2000a:60150.

[DM] S.W. Drury and M.R. Murty (eds.), Harmonic Analysis and Number Theory, Papers in honour of Carl S. Herz (Montreal, 1996), CMS Conf. Proc., 21, Amer. Math. Soc., Providence, 1997; MR 98d:00028.

[D] D. Dufresne, The distribution of a perpetuity, with applications to risk theory and pension funding, Scand. Actuar. J., (1990), 39-79; MR 92i:62195.

[E] S.N. Evans, Local field Gaussian measures, Seminar on Stochastic Processes (Gainesville, 1988) (E. Çinlar et al., eds.), Progr. Probab., 17, Birkhäuser, Boston, (1989), 121-160; MR 91e:60121.

[F1] A. Figà-Talamanca, An application of Gelfand pairs to a problem of diffusion in compact ultrametric spaces, Notes of a lecture delivered at the seminar "Topics in probability theory and Lie groups: Boundary theory" (Montreal, September 1992).

[F2] _ Diffusion on compact ultrametric spaces, Noncompact Lie Groups and Some of Their Applications (San Antonio, 1993) (E.A. Tanner, R. Wilson, eds.), NATO Adv. Sci. Inst. Ser. C Math. Phys. Sci., 429, Kluwer, Dordrecht, (1994), 157-167; MR 95m:60012.

[F3] _ Local fields and trees, Harmonic Functions on Trees and Buildings, (New York, 1995) (A. Korányi, ed.), Contemp. Math., 206, Amer. Math. Soc., Providence, (1997), 3-16; MR 98h:22005.

[GK] B.V. Gnedenko and A.N. Kolmogorov, Limit Distributions for Sums of Independent Random Variables, Addison-Wesley Math. Ser., Addison-Wesley, Reading, 1968; MR 38 \#1722.

[GR] I.S. Gradshteyn and I.M. Ryzhik, Table of Integrals, Series, and Products, Academic Press, Boston, 1994; MR 94g:00008.

[H] C. Hassenforder, Les lois de Cauchy sur les bouts de l'arbre homogène, Probab. Theory Related Fields, 78 (1988), 603-616; MR 90a:60012.

[I] R.S. Ismagilov, Spectrum of a self-adjoint operator in $L_{2}(K)$, where $K$ is a local field; analog of the Feynman-Kac formula, Theoret. and Math. Phys., 89 (1991), 1024-1028; MR 92m:47049.

[K] A.N. Kochubei, Limit theorems for sums of p-adic random variables, Exposition. Math., 16 (1998), 425-439; MR 99m:60016. 
[L] S. Lang, $S L_{2}(\mathbf{R})$, Grad. Texts in Math., 105, Springer, New York, 1985; MR $\mathbf{8 6 \mathbf { j }}: 22018$.

[ST] G. Samorodnitsky and M.S. Taqqu, Stable Non-Gaussian Random Processes: Stochastic Models with Infinite Variance, Stochastic Model., Chapman \& Hall, New York, 1994; MR 95f:60024.

[T] M.H. Taibleson, Fourier Analysis on Local Fields, Math. Notes, 15, Princeton Univ. Press, Princeton, 1975; MR 58 \#6943.

[V] V.S. Varadarajan, Path integrals for a class of p-adic Schrödinger equations, Lett. Math. Phys., 39 (1997), 97-106; MR 98m:81083.

[W] A. Weil, Basic Number Theory, Classics in Math., Springer, Berlin, 1995; MR 96c:11002.

[Y] M. Yor, Sur certaines fonctionnelles exponentielles du mouvement brownien réel, J. Appl. Probab., 29 (1992), 202-208; MR 93g:60179.

Received May 5, 1999.

Dipartimento di Matematica

UNIVERSITÀ DI ROMA "TOR VERGATA"

Via Della Ricerca Scientifica

00133 Roma

ITALY

E-mail address: baldi@mat.uniroma2.it

Dipartimento di Matematica "G. Castelnuovo"

UNIVERSità DI ROMA "LA SAPIENZA"

Piazzale A. Moro 2

00185 Roma

ITALY

E-mail address: casadio@science.unitn.it

Dipartimento di Matematica "G. Castelnuovo"

Università Di Roma "LA SAPIEnZA"

Piazzale A. Moro 2

00185 RoMA

ITALY

E-mail address: sandroft@mat.uniroma1.it 